\title{
Membrane division, restricted membrane creation and object complexity in P systems
}

\author{
ARTIOM ALHAZOVa, RUDOLF FREUNDb and AGUSTÍN RISCOS-NÚÑEZc
}

a Institute of Mathematics and Computer Science, Academy of Sciences of Moldova,

Str. Academiei 5, Chişinău, MD 2028, Moldova

bResearch Group on Mathematical Linguistics, Rovira i Virgili University,

Pl. Imperial Tàrraco 1, 43005 Tarragona, Spain

aFaculty of Informatics, Vienna University of Technology, Favoritenstr. 9, A-1040 Vienna, Austria cResearch Group on Natural Computing, Department of Computer Science and Artificial Intelligence,

University of Sevilla, Avda. Reina Mercedes s/n, 41012 Sevilla, Spain

\begin{abstract}
We improve, by using register machines, some existing universality results for specific models of $\mathrm{P}$ systems. P systems with membrane creation are known to generate all recursively enumerable sets of vectors of non-negative integers, even when no region (except the environment) contains more than one object of the same kind. We show here that they generate all recursively enumerable languages, and that two membrane labels are sufficient (the same result holds for accepting all recursively enumerable vectors of non-negative integers). Moreover, at most two objects are present inside the system at any time in the generative case. We then prove that $10+m$ symbols are sufficient to generate any recursively enumerable language over $m$ symbols. $\mathrm{P}$ systems with active membranes without polarizations are known to generate all recursively enumerable sets of vectors of non-negative integers. We show that they generate all recursively enumerable languages; four starting membranes with three labels or seven starting membranes with two labels are sufficient. P systems with active membranes and two polarizations are known to generate/accept all recursively enumerable sets of vectors of non-negative integers, using only rules of rewriting and sending objects out. We show that accepting can be done by deterministic systems. Finally, we show that $\mathrm{P}$ systems with restricted membrane creation (the newly created membrane can only be of the same kind as the parent one) generate at least matrix languages, even when having at most one object in the configuration (except the environment). We conclude by presenting a summary of the main results obtained in this paper and a list of open questions.
\end{abstract}

Keywords: Membrane division; Restricted membrane creation; Object complexity; P systems

\section{Introduction}

P systems with symbol objects is a theoretical framework for distributed parallel multiset processing, initiated by Păun in 1998 [1]. A systematic field survey can be found in [2], and [3] contains a comprehensive bibliography. 
The aim of this article is to improve the descriptive complexity parameters or properties of a few universality results. More precisely, we shall speak about object complexity (bounds in the starting configuration, in any configuration, in the alphabet) and also about membrane complexity.

Let us denote the set of all recursively enumerable sets of ( $k$-dimensional) vectors of non-negative integers by $P s R E$ ( $P S R E(k)$, respectively), whereas the set of all recursively enumerable languages (over a $k$-letter alphabet) is denoted by $R E(R E(k)$, respectively).

It has been shown [4] that $\mathrm{P}$ systems with membrane creation generate PsRE, even when every region (except the environment) contains at most one object of every kind, but using an unbounded number of membrane labels. We will show that $R E$ is generated using only two membrane labels and at most two objects present inside the system throughout any computation. The accepting case is also considered and, again, two membrane labels are sufficient. On the other hand, by using an unbounded membrane alphabet we can bound the symbol alphabet by $10+m$ objects, where $m$ is the size of the output alphabet.

We also know from [4] that $\mathrm{P}$ systems with active membranes without polarization generate $P s R E$, again working with an unbounded number of membranes. We will show that $R E$ is generated by $\mathrm{P}$ systems with four membranes and three labels or seven membranes with two labels in the initial configuration.

As shown in [5, 6], P systems with two polarizations and rules of types (a) - rewriting —and (c)—sending an object out—generate PsRE using two membranes or accept PsRE using one membrane. In this article we will show that deterministic systems of this kind with one membrane accept PsRE.

Returning to the membrane creation case, we investigate the restriction of the model to creating membranes only of the same kind as the parent membrane.

\section{Definitions}

After some preliminary definitions, we recall the basic facts concerning register machines and matrix grammars (without appearance checking) and give the necessary definitions for the specific models of the $\mathrm{P}$ systems considered in this paper.

\subsection{Preliminaries}

The set of non-negative integers is denoted by $\mathbb{N}$. An alphabet $V$ is a finite non-empty set of abstract symbols. Given $V$, the free monoid generated by $V$ under the operation of concatenation is denoted by $V^{*}$; the empty string is denoted by $\lambda$, and $V^{*}-\{\lambda\}$ is denoted by $V^{+}$. By $|x|$ we denote the length of the string $x$ over $V$.

For more details as well as basic results from the theory of formal languages, the reader is referred to $[7,8]$.

\subsection{Register machines}

A register machine is a construct $M=\left(m, P, l_{0}, l_{h}\right)$, where $m$ is the number of registers, $P$ is a finite set of instructions injectively labeled with elements from a given set $l a b(M), l_{0}$ is the initial/start label, and $l_{h}$ is the final label.

The instructions are of the following forms.

$-l:\left(A(r), l^{\prime}, l^{\prime \prime}\right)$ 
Add 1 to the contents of register $r$ and proceed to the instruction (labeled with) $l^{\prime}$ or $l^{\prime \prime}$ (an ADD instruction). For deterministic machines, we require $l^{\prime}=l^{\prime \prime}$.

$-l:\left(S(r), l^{\prime}, l^{\prime \prime}\right)$

If register $r$ is not empty, then subtract 1 from its contents and go to instruction $l^{\prime}$, otherwise proceed to instruction $l^{\prime \prime}$ (a conditional SUB instruction).

$-l_{h}:$ halt

Stop the machine. (The final label $l_{h}$ is only assigned to this instruction.)

When considering the generation of languages, we use the model of a register machine with output tape, which also uses a tape operation:

$-l:\left(\right.$ write $\left.(a), l^{\prime \prime}\right)$

Write symbol $a$ on the output tape and go to $l^{\prime \prime}$.

We then also specify the output alphabet $T$ in the description of the register machine with output tape, i.e. we write $M=\left(m, T, P, l_{0}, l_{h}\right)$.

The following results are folklore (e.g., see [9]).

Proposition 2.1 Let $L \subseteq N^{m}$ be a recursively enumerable set of (vectors of) non-negative integers. Then $L$ can be generated/accepted by a register machine/deterministic register machine with at most $m+2$ registers; moreover, at the beginning/at the end of a computation, all registers are empty; the result/the input of a computation appears in the first $m$ registers.

Let $L \subseteq V^{*}$ be a recursively enumerable language. Then $L$ can be generated by a register machine with output tape with two registers.

\subsection{Matrix grammars}

A context-free matrix grammar (without appearance checking) is a construct $G=$ $(N, T, S, M)$, where $N$ and $T$ are sets of non-terminal and terminal symbols, respectively, with $N \cap T=\emptyset, S \in N$ is the start symbol, $M$ is a finite set of matrices, $M=\left\{m_{i} \mid 1 \leq i \leq n\right\}$, where the matrices $m_{i}$ are sequences of the form $m_{i}=\left(m_{i, 1}, \ldots, m_{i, n_{i}}\right), n_{i} \geq 1,1 \leq i \leq n$, and the $m_{i, j}, 1 \leq j \leq n_{i}, 1 \leq i \leq n$, are context-free productions over $(N, T)$.

For $m_{i}=\left(m_{i, 1}, \ldots, m_{i, n_{i}}\right)$ and $v, w \in(N \cup T)^{*}$ we define $v \Longrightarrow_{m_{i}} w$ if and only if there are $w_{0}, w_{1}, \ldots, w_{n_{i}} \in(N \cup T)^{*}$ such that $w_{0}=v, w_{n_{i}}=w$, and for each $j, 1 \leq j \leq n_{i}, w_{j}$ is the result of the application of $m_{i, j}$ to $w_{j-1}$. The language generated by $G$ is

$$
\begin{aligned}
L(G)= & \left\{w \in T^{*} \mid S \Longrightarrow_{m_{i_{1}}} w_{1} \cdots \Longrightarrow_{m_{i_{k}}} w_{k}=w, w_{j} \in(N \cup T)^{*},\right. \\
& \left.m_{i_{j}} \in M \text { for } 1 \leq j \leq k, k \geq 1\right\} .
\end{aligned}
$$

The family of languages generated by matrix grammars without appearance checking is denoted by $M A T^{\lambda}$. It is known that, for the family of Parikh sets of languages generated by matrix grammars $P S M A T^{\lambda}$, we have $P s M A T^{\lambda} \subset P S R E$. Further details concerning matrix grammars can be found in $[7,8]$. We only mention that the power of matrix grammars is not reduced if we only work with matrix grammars in the $f$-binary normal form where $N$ is the disjoint union of $N_{1}, N_{2}$, and $\{S, f\}$ and $M$ contains rules of the following forms:

(i) ( $S \rightarrow X_{\text {init }} A_{\text {init }}$ ), with $X_{\text {init }} \in N_{1}, A_{\text {init }} \in N_{2}$;

(ii) $(X \rightarrow Y, A \rightarrow x)$, with $X \in N_{1}, Y \in N_{1} \cup\{f\}, A \in N_{2}$, and $x \in\left(N_{2} \cup T\right)^{*},|x| \leq 2$;

(iii) $(f \rightarrow \lambda)$. 
Moreover, there is only one matrix of type 1, which is only used in the first step of any derivation, and only one matrix of type 3 , which is only used in the last step of a derivation yielding a terminal result.

\subsection{P systems}

The first model of $\mathrm{P}$ systems we consider is that of $P$ systems with non-cooperative multisetrewriting rules (ncoo) with specifying targets (tar), also using membrane creation (mcre) and membrane dissolution $(\delta$ ) possibilities. Such a system (of initial degree $m \geq 1$ ) is of the form

$$
\Pi=\left(O, H, \mu, w_{1}, \ldots, w_{m}, R_{1}, \ldots, R_{n}\right),
$$

where $O$ is the alphabet of objects, $H$ is the set of labels for membranes (we assume here that $H$ contains $n$ labels), $\mu$ is the initial membrane structure, consisting of $m$ membranes labeled (not necessarily in a one-to-one manner) with elements of $H, w_{1}, \ldots, w_{m}$ are strings over $O$ representing the multisets of objects present in the $m$ compartments (also called regions) of $\mu$, and $R_{1}, \ldots, R_{n}$ are the (finite) sets of rules associated with the $n$ labels from $H$. These rules can be of the form (1) $a \rightarrow v$ and (2) $a \rightarrow\left[{ }_{i} b\right]_{i}$, where $v \in O^{*}, a, b \in O, i \in H$, and either $v \in(O \times \text { tar })^{*}$ or $v \in(O \times \operatorname{tar})^{*}\{\delta\}$, with tar $=\{$ here, out $\} \cup\left\{\right.$ in $\left._{j} \mid j \in H\right\}$. The presence of $\delta$ on the right-hand side of a rule means that the application of the rule leads to the dissolution of the membrane.

The meaning of a rule of type (1) is that the object $a$ from the region associated with the rule 'reacts', and as a result the objects specified by $v$ are produced. The objects from $v$ have associated target commands, of the form here, out, $\mathrm{in}_{j}$, which specify where the object should be placed: here means that the object remains in the region where it is produced, $i n_{j}$ means that it has to go to membrane $j$, provided that it is directly inside the membrane where the rule is applied (otherwise the rule cannot be used), and out indicates that the object should exit the current membrane, going to the surrounding region-which is the environment in the case of the skin membrane of the system. In general, the indication here is not explicitly written. If the special symbol $\delta$ is present, this means that, after using the rule, the membrane is dissolved, and all its contents, objects and membranes alike, become elements of the surrounding region. The skin membrane is never dissolved. A rule $a \rightarrow\left[{ }_{i} b\right]_{i}$ of type (2) means that object $a$ produces a new membrane, with label $i$, containing the object $b$.

In this article we will also consider $\mathrm{P}$ systems with restricted membrane creation (we will denote this feature by mcre $_{r}$ ): $a \rightarrow\left[{ }_{i} b\right]_{i} \in R_{i}$, meaning that, in region $i$, it is only possible to create membranes with label $i$.

Knowing the label of the membrane, we also know the rules associated with it. We recall that the number of membrane labels (i.e. kinds of membranes) is $n$ and the rules are associated with the membrane labels, while the number of membranes initially is $m$ and can change during the computation. The rules are used in the non-deterministic (the objects and the rules are chosen non-deterministically) maximally parallel way (no further object can evolve after having chosen the objects for the rules), thus obtaining transitions between a configuration of the system to another one.

$P$ systems with active membranes with $k$ polarizations (active $e_{k}$ ) are constructs

$$
\Pi=\left(O, H, E, \mu, w_{1}, \ldots, w_{m}, R\right),
$$

with the components $O, H, \mu, w_{1}, \ldots, w_{m}$ defined in the same way as above, with membranes of $\mu$ also having associated polarizations from $E$ (having cardinality $k$ ) and with the rules from $R$ of the following forms: (a) $[a \rightarrow v]_{h}^{e}$-rewriting-like, (b) $a[]_{h}^{e} \rightarrow[b]_{h}^{e^{\prime}}$ 
and (c) $[a]_{h}^{e} \rightarrow[]_{h}^{e^{\prime}} b$-bring an object inside the membrane/send an object out of the membrane (possibly changing its polarization), (d) $[a]_{h}^{e} \rightarrow b$-dissolve the membrane, producing another object (the contents of the dissolved membrane are released into the surrounding region), and (e) $[a]_{h}^{e} \rightarrow[b]_{h}^{e^{\prime}}[c]_{h}^{e^{\prime \prime}}$-membrane division, where two membranes with the same label (but possibly different polarizations) are produced, each containing a new object (all other objects are duplicated). In all cases, $a, b, c \in O, v \in O^{*}, e, e^{\prime}, e^{\prime \prime} \in E$ and $h \in H$.

The rules of type (a) are applied in the maximally parallel way, whereas at most one rule of types (b), (c), (d) and (e) can be applied for each membrane at any step of the computation. If we have only one polarization, we omit specifying it (and in the literature, the subscript 0 is then usually added to the types of rules defined above, thus yielding $\left(a_{0}\right)$, etc.).

In both models, when a configuration is reached where no rule can be applied, the computation stops, and the multiplicity of the objects sent into the environment during the computation is said to be computed by the system along that computation. We denote by $P s(\Pi)$ the set of vectors generated in this way (by means of all computations) by a system $\Pi$. If we take into account the sequence of symbols as they are sent out into the environment (when two or more objects leave the system at the same moment, then all permutations of these objects are considered), then we obtain the string language generated by $\Pi$, which is denoted by $L(\Pi)$. When considering $\Pi$ as an accepting system for a set of vectors, we put the input multiset into the skin membrane and accept by halting computations.

By $X O_{n_{1}, n_{2}, n_{3}} P_{n_{4}, n_{5}, n_{6}} F$ we denote the resulting families generated by such $\mathrm{P}$ systems, where: (1) $X$ is either $L$ for languages or $P s$ for sets of vectors of non-negative integers; we add the subscript $a$ when considering accepting systems; (2) $F$ is the list of features used in the model (e.g., we consider (ncoo, tar, mcre, $\delta)$, (ncoo, tar, mcre $\left._{r}, \delta\right),\left(\right.$ active $\left._{1}, a, b, c, d, e\right)$, and (active $2, a, c)$ ); (3) the numbers $n_{4}, n_{5}, n_{6}$ represent the bounds on the starting number of membranes, the maximal number of membranes in any computation, and the number of membrane labels, $*$ representing the absence of a bound (if all three numbers are $*$, then we simply omit them); and (4) the numbers $n_{1}, n_{2}, n_{3}$ have the same meaning, but for the objects inside the system; the middle parameter, $n_{2}$ or $n_{5}$, can be replaced by $n_{2}^{\prime} / n_{2}$ or $n_{5}^{\prime} / n_{5}$, where the primed numbers indicate the bounds on the number of objects or membranes ever present in the system during halting computations only, thus refining this complexity measure.

\section{Membrane creation}

\subsection{Generating}

The first theorem shows how recursively enumerable languages can be generated by $\mathrm{P}$ systems with a small number of objects inside the system and a small number of membrane labels.

TheOREM $3.1 \quad L O_{1,2, *} P_{1, *, 2}($ ncoo, tar, mcre,$\delta)=R E$.

Proof Due to proposition 2.1, we construct a $\mathrm{P}$ system simulating a register machine $M=$ $\left(2, T, P, l_{0}, l_{h}\right)$ with output tape and two registers; $P_{-}$denotes the set of all SUB instruction labels.

$$
\begin{aligned}
\Pi & =\left(O, H,[1]_{1}, w_{1}, R_{1}, R_{2}\right), \\
O & =T \cup\left\{a_{1}, a_{2}, C_{1}, C_{2}, g_{0}, g_{1}, g_{2}, t\right\} \cup P \cup\left\{l_{1}, l_{2}, l_{3}, l_{4}, l_{5}, l_{6}, l_{7} \mid l \in P_{-}\right\}, \\
H & =\{1,2\}, \\
w_{1} & =g_{0},
\end{aligned}
$$




$$
\begin{aligned}
& R_{1}=R_{1, I} \cup R_{1, A} \cup R_{1, S} \cup R_{1, D} \cup R_{1, Z} \cup R_{1, O}, \\
& R_{2}=R_{2, I} \cup R_{2, A} \cup R_{2, S} \cup R_{2, D} \cup R_{2, Z} .
\end{aligned}
$$

For clarity, the rules are grouped into categories (initialization, add, subtract, decrement case, zero case, output).

\section{Initialization:}

$$
\begin{aligned}
& R_{1, I}=\left\{g_{0} \rightarrow\left[2 g_{0}\right]_{2}, g_{1} \rightarrow\left[\begin{array}{ll}
1 & g_{2}
\end{array}\right]_{1}, g_{2} \rightarrow\left(l_{0}\right)_{\text {out }}\right\} \\
& R_{2, I}=\left\{g_{0} \rightarrow\left(g_{1}\right)_{\text {out }}\right\}
\end{aligned}
$$

\section{Output:}

$$
R_{1, O}=\left\{l \rightarrow l^{\prime} a_{\text {out }} \mid a \in T, l:\left(\text { write }(a), l^{\prime}\right) \in P\right\} .
$$

Add:

$$
\begin{aligned}
R_{1, A}= & \left\{l \rightarrow l^{\prime}\left(C_{2}\right)_{i n_{i}}, l \rightarrow l^{\prime \prime}\left(C_{2}\right)_{i n_{i}} \mid l:\left(A(i), l^{\prime}, l^{\prime \prime}\right) \in P, i \in\{1,2\}\right\} \\
& \cup\left\{C_{2} \rightarrow\left[{ }_{2} t\right]_{2}, t \rightarrow \lambda\right\}, \\
R_{2, A}= & \left\{C_{2} \rightarrow\left[{ }_{2} t\right]_{2}, t \rightarrow \lambda\right\} .
\end{aligned}
$$

\section{Subtract:}

$$
\begin{aligned}
R_{1, S}= & \left\{l \rightarrow\left(l_{1} C_{1}\right)_{i n_{i}} \mid l:\left(S(i), l^{\prime}, l^{\prime \prime}\right) \in P, i \in\{1,2\}\right\} \\
& \left.\cup\left\{C_{1} \rightarrow{ }_{1} t\right]_{1}\right\} \cup\left\{l_{1} \rightarrow\left(l_{2}\right)_{i n_{2}} \mid l:\left(S(1), l^{\prime}, l^{\prime \prime}\right) \in P\right\}, \\
R_{2, S}= & \left\{C_{1} \rightarrow\left[{ }_{1} t\right]_{1}\right\} \cup\left\{l_{1} \rightarrow\left(l_{2}\right)_{i n_{2}} \mid l:\left(S(2), l^{\prime}, l^{\prime \prime}\right) \in P\right\} .
\end{aligned}
$$

\section{Decrement case:}

$$
\begin{aligned}
R_{1, D}= & \left\{l_{4} \rightarrow l_{5} \delta \mid l \in P_{-}\right\} \\
& \cup\left\{l_{3} \rightarrow\left(l_{4}\right)_{\text {in }}, l_{5} \rightarrow\left(l^{\prime}\right)_{\text {out }} \mid l:\left(S(1), l^{\prime}, l^{\prime \prime} \in P\right)\right\}, \\
R_{2, D}= & \left\{l_{2} \rightarrow l_{3} \delta \mid l \in P_{-}\right\} \\
& \cup\left\{l_{3} \rightarrow\left(l_{4}\right)_{\text {in }}, l_{5} \rightarrow\left(l^{\prime}\right)_{\text {out }} \mid l:\left(S(2), l^{\prime}, l^{\prime \prime} \in P\right)\right\} .
\end{aligned}
$$

\section{Zero case:}

$$
\begin{aligned}
R_{1, Z}= & \left\{l_{6} \rightarrow l_{7} \delta \mid l \in P_{-}\right\} \\
& \cup\left\{l_{1} \rightarrow\left(l_{6}\right)_{\text {in }}, l_{7} \rightarrow\left(l^{\prime \prime}\right)_{\text {out }} \mid l:\left(S(1), l^{\prime}, l^{\prime \prime} \in P\right)\right\}, \\
R_{2, Z}= & \left\{l_{1} \rightarrow\left(l_{6}\right)_{\text {in }}, l_{7} \rightarrow\left(l^{\prime \prime}\right)_{\text {out }} \mid l:\left(S(2), l^{\prime}, l^{\prime \prime} \in P\right)\right\} .
\end{aligned}
$$

Initially, by means of the auxiliary objects $g_{i}$, we create two membranes inside the skin region, labeled 1 and 2 , respectively. These membranes will be referred to as cluster membranes (because they will contain inside them a number of elementary membranes). We finish the initialization phase by generating an object $l_{0}$ in the skin region.

The values of the two registers $i, i \in\{1,2\}$, are represented by the number of elementary membranes labeled 2 that occur inside the corresponding cluster membrane $i$. The duty of the object $C_{i}$ is to create membrane $i$. Object $t$ is not needed for the computation, it is only used 
to keep the usual form of the membrane creation rules and is immediately erased after having been created.

Writing an output symbol $a \in T$ is done by a non-cooperative rule changing the instruction label and producing a symbol $a$ that is immediately sent out. To increment a register, a membrane labeled 2 is created inside the corresponding cluster membrane.

In order to simulate a subtraction on register $i$ we send the objects $l_{1}$ and $C_{1}$ into the cluster membrane $i$ and then proceed in the following way: while creating a membrane with label 1 , object $l_{1}$ tries to enter some membrane with label 2 as $l_{2}$. If such a membrane exists (i.e. register $i$ is not empty), then $l_{2}$ changes to $l_{3}$ and dissolves the membrane, thus being spilled back into the cluster membrane. Before proceeding to the next label, we have to get rid of the auxiliary membrane 1 that was created inside the cluster membrane by $C_{1}$. To this end, $l_{3}$ enters into membrane 1 as $l_{4}$ and dissolves it, thereby changing to $l_{5}$. Finally, $l_{5}$ sends an object $l^{\prime}$ out to the skin region. As an overall result, $l$ has been replaced by $l^{\prime}$ and the number of membranes with label 2 inside the cluster membrane $i$ has been reduced by one. If, on the other hand, no membrane with label 2 exists in the cluster membrane, then $l_{1}$ waits for one step and then enters the newly created membrane 1 as $l_{6}$. Immediately afterwards, it changes to $l_{7}$ and dissolves the membrane. Finally, $l_{7}$ sends out an object $l^{\prime \prime}$ into the skin region. As an overall result, in the absence of membranes with label 2 inside the cluster membrane $i, l$ has been replaced by $l^{\prime \prime}$.

Notice that, inside the system, there can never be more than one copy of the same object. In fact, the number of objects inside the system never exceeds two (it can only be two after the first step of an ADD or SUB instruction).

\subsection{Accepting}

Notice that the simulation of the register machine instructions in theorem 3.1 is deterministic (the non-determinism arises from the non-determinism of the register machine program itself, not from the simulation). For the case of accepting sets of vectors in a deterministic way, we also specify the cardinality of the input alphabet, i.e. the number of components in the (Parikh) vectors.

THEOREM $3.2 \operatorname{DPs}_{a}(m) O P_{1, *, 2}($ ncoo, tar, mcre, $\delta)=\operatorname{PsRE}(m)$.

Proof Given a recursively enumerable set of vectors of non-negative integers, we now simulate a register machine $M=\left(m+2, P, l_{0}, l_{h}\right)$ (see proposition 2.1); the input vector is represented in the skin by the corresponding numbers of symbols $\left(a_{i}, i\right), 1 \leq i \leq m$, for the $i$ th component.

$$
\begin{aligned}
\Pi= & \left(O, H,[1]_{1}, w_{1}, R_{1}, R_{2}\right), \\
O= & \left\{\left(a_{i}, j\right) \mid 1 \leq i \leq m+2,1 \leq j \leq i\right\} \\
& \cup\left\{C_{1}, C_{2}, t\right\} \cup\left\{g_{i} \mid 0 \leq i \leq 4(m+2)\right\} \\
& \cup P \cup\left\{l_{1}, l_{2}, l_{3}, l_{4}, l_{5}, l_{6}, l_{7} \mid l \in P_{-}\right\} \\
& \cup\left\{(l, k, j) \mid l \in P_{-}, 0 \leq k \leq 2,1 \leq j \leq m+2\right\}, \\
H= & \{1,2\}, \\
w_{1}= & g_{0}, \\
R_{1}= & R_{1, I} \cup R_{1, A} \cup R_{1, S} \cup R_{1, D} \cup R_{1, Z}, \\
R_{2}= & R_{2, I} \cup R_{2, A} \cup R_{2, S} \cup R_{2, D} \cup R_{2, Z} .
\end{aligned}
$$


Theorem 3.1

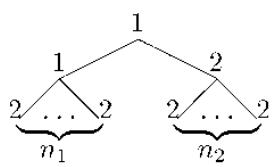

Theorem 3.3

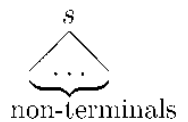

Theorem 3.2

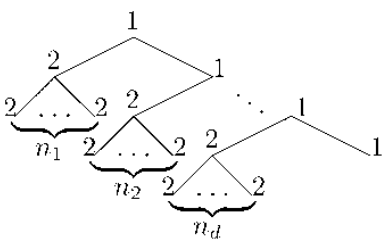

Theorems 3.4. 3.5

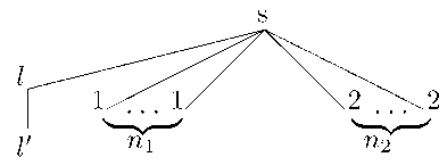

Figure 1. Membrane structures for the membrane creation proofs.

Like in the previous theorem, we list the rules by categories (initialization, add, subtract, decrement case, zero case). We again start with the object $g_{0}$, which now starts the creation of the membrane structure $\left(\left[\left[_{1}[2]_{2}\right)^{m+2}(]_{1}\right)^{m+2}\right.$, where each membrane with label 2 (cluster membrane) corresponds to a register. In parallel, the input objects enter the corresponding membranes and become membranes with label 2; see figure 1.

\section{Initialization:}

$$
\begin{aligned}
R_{1, I}= & \left\{g_{3 i} \rightarrow\left[2 g_{3 i+1}\right]_{2}, g_{3 i+2} \rightarrow\left[1 g_{3 i+3}\right]_{1},\right. \\
& \left.g_{3(m+2)+i} \rightarrow\left(g_{3(m+2)+i+1}\right)_{\text {out }} \mid 0 \leq i \leq m+1\right\} \\
& \cup\left\{g_{4(m+2)} \rightarrow l_{0}\right\} \\
& \cup\left\{\left(a_{i}, j\right) \rightarrow\left(a_{i}, j-1\right)_{i n_{1}} \mid 1 \leq i \leq m, 2 \leq j \leq i\right\} \\
& \cup\left\{\left(a_{i}, 1\right) \rightarrow\left(C_{2}\right)_{\text {in }_{2}} \mid 1 \leq i \leq m\right\}, \\
R_{2, I}= & \left\{g_{3 i+1} \rightarrow\left(g_{3 i+2}\right)_{\text {out }} \mid 0 \leq i \leq m+1\right\} \cup\left\{C_{2} \rightarrow\left[{ }_{2} t\right]_{2}, t \rightarrow \lambda\right\} .
\end{aligned}
$$

We then perform a deterministic simulation of the instructions in the program of the deterministic accepting register machine $M$. We now list the rules for ADD instructions (registers $m+1, m+2$ for the simulation of the working registers; observe that, for the input initialization of the registers $i, 1 \leq i \leq m$, similar rules for the corresponding symbols $\left(a_{i}, i\right)$ are used).

Add:

$$
\begin{aligned}
R_{1, A}= & \left\{l \rightarrow l^{\prime}\left(a_{i}, i\right), l \rightarrow l^{\prime \prime}\left(a_{i}, i\right),\right. \\
& \left(a_{i}, j\right) \rightarrow\left(a_{i}, j-1\right)_{i n_{1}},\left(a_{i}, 1\right) \rightarrow\left(C_{2}\right)_{i n_{2}} \mid \\
& \left.l:\left(A(i), l^{\prime}, l^{\prime \prime}\right) \in P, m+1 \leq i \leq m+2,2 \leq j \leq i\right\}, \\
R_{2, A}= & \left\{C_{2} \rightarrow[2 t]_{2}, t \rightarrow \lambda\right\} .
\end{aligned}
$$

The main reason for the additional rules is that we have to go to that level of the membrane structure that corresponds to the register affected by the instruction. The same has to be done for the SUB instructions. 


\section{Subtract:}

$$
\begin{aligned}
R_{1, S}= & \left\{l \rightarrow(l, 0, i),(l, 0,1) \rightarrow\left(l_{1} C_{1}\right)_{i n_{2}} \mid l:\left(S(i), l^{\prime}, l^{\prime \prime}\right) \in P\right\} \\
& \cup\left\{(l, 0, j) \rightarrow(l, 0, j-1)_{i n_{1}} \mid 2 \leq j \leq i, l:\left(S(i), l^{\prime}, l^{\prime \prime}\right) \in P\right\} \\
& \cup\left\{(l, k, j) \rightarrow(l, k, j-1)_{\text {out }} \mid 2 \leq j \leq i, 1 \leq k \leq 2,\right. \\
& \left.l:\left(S(i), l^{\prime}, l^{\prime \prime}\right) \in P\right\} \\
& \cup\left\{(l, 1,1) \rightarrow l^{\prime},(l, 2,1) \rightarrow l^{\prime \prime} \mid l:\left(S(i), l^{\prime}, l^{\prime \prime}\right) \in P\right\} \cup\{t \rightarrow \lambda\}, \\
R_{2, S}= & \left\{l_{1} \rightarrow\left(l_{2}\right)_{i n_{2}}, C_{1} \rightarrow\left[{ }_{1} t\right]_{1}\right\} .
\end{aligned}
$$

The decrement case and the zero case are handled very much as in theorem 3.1 for register 2 .

\section{Decrement case:}

$$
\begin{aligned}
R_{1, D}= & \left\{l_{4} \rightarrow l_{5} \delta \mid l \in P_{-}\right\} \\
R_{2, D}= & \left\{l_{2} \rightarrow l_{3} \delta \mid l \in P_{-}\right\} \\
& \cup\left\{l_{3} \rightarrow\left(l_{4}\right)_{\text {in }}, l_{5} \rightarrow(l, 1, i)_{\text {out }} \mid l:\left(S(i), l^{\prime}, l^{\prime \prime} \in P\right)\right\} .
\end{aligned}
$$

\section{Zero case:}

$$
\begin{aligned}
& R_{1, Z}=\left\{l_{6} \rightarrow l_{7} \delta \mid l \in P_{-}\right\} \\
& R_{2, Z}=\left\{l_{1} \rightarrow\left(l_{6}\right)_{\text {in }}, l_{7} \rightarrow(l, 2, i)_{\text {out }} \mid l:\left(S(i), l^{\prime}, l^{\prime \prime} \in P\right)\right\}
\end{aligned}
$$

After the correct simulation of the decrement case or the zero case, the symbol $(l, 1, i)$ or the symbol $(l, 2, i)$, respectively, is released; by decrementing the third component, these symbols can travel along the line of membranes labeled 1 back to the skin membrane, where the rule $(l, 1,1) \rightarrow l^{\prime}$ or the rule $(l, 2,1) \rightarrow l^{\prime \prime}$ is applied; hence, after decrementing, the label $l^{\prime}$ is obtained, whereas in the zero case we continue with label $l^{\prime \prime}$.

We finally observe that all the derivations in $\Pi$ are performed deterministically, which completes the proof.

\subsection{Generating with one object}

Considering P systems having only one object inside the system during the whole computation, we realize that such $\mathrm{P}$ systems with one object work in a sequential way, hence the following holds.

THEOREM $3.3 \quad P S M A T^{\lambda}$ is characterized by $P$ systems

$$
\Pi=\left(O, H, \mu, w_{1}, \ldots, w_{m}, R_{1}, \ldots, R_{n}\right),
$$

where (a) the initial membrane structure is limited by two levels (any membrane inside the skin is elementary) and label 1 of the skin membrane is unique (i.e. the labels of the other membranes inside are different from 1$) ;(b)$ exactly one of the multisets $w_{i}$ consists of exactly one object, whereas all the other initial multisets are empty; $(c)$ the rules in $R_{1}$ are of the form $a \rightarrow b u_{\text {out }}, a \rightarrow b_{\text {in }_{i}} u_{\text {out }}, a \rightarrow b$, or $a \rightarrow\left[{ }_{i} b\right]_{i}$ with $a, b \in O, u \in O^{*}$, and $i \in H^{\prime}$, where $H^{\prime}=H-\{s\}$; and $(d)$ the rules in $R_{i}, 2 \leq i \leq n$, are of the form $a \rightarrow b, a \rightarrow b_{\text {out }}$, or $a \rightarrow b \delta$. 
Proof Let $\Pi$ be a $\mathrm{P}$ system obeying the conditions given above. We first observe that, due to the form of the initial configuration as well as due to the restricted forms of the rules, during any computation: (i) the membrane structure is limited by two levels (any membrane inside the skin is elementary); and (ii) the number of symbols inside the system is exactly one. Therefore, when constructing a matrix grammar $G=(N, T, S, M)$ simulating $\Pi$, with $T \subseteq O$, a membrane $i$ can be represented by the non-terminal $i$, while the object $a$ and its position in membrane $i$ can be stored as a pair $(a, i)$. With InCon denoting the set of representations of the initial configuration given by $\mu, w_{1}, \ldots, w_{m}$ (in fact, only one such representation is necessary), we can now specify the matrix grammar $G$ as follows:

$$
\begin{aligned}
N= & H \cup O \times H \cup\{\overline{(a, i)} \mid(a, i) \in F\}, \\
M= & \{(S \rightarrow w) \mid w \in I n C o n\} \\
& \cup\left\{((a, s) \rightarrow(b, s) u) \mid a \rightarrow b u_{\text {out }} \in R_{s}\right\} \\
& \cup\left\{((a, s) \rightarrow(b, i) u, i \rightarrow i) \mid a \rightarrow b_{\text {in }} u_{\text {out }} \in R_{s}, i \in H^{\prime}\right\} \\
& \cup\left\{((a, i) \rightarrow(b, i)) \mid a \rightarrow b \in R_{i}, i \in H\right\} \\
& \cup\left\{((a, i) \rightarrow(b, s)) \mid a \rightarrow b_{\text {out }} \in R_{i}, i \in H^{\prime}\right\} \\
& \cup\left\{((a, s) \rightarrow(b, i) i) \mid a \rightarrow[i b]_{i} \in R_{s}, i \in H^{\prime}\right\} \\
& \cup\left\{((a, i) \rightarrow(b, s), i \rightarrow \lambda) \mid a \rightarrow b \delta \in R_{i}, i \in H^{\prime}\right\} \\
& \cup\{((a, i) \rightarrow \overline{(a, i)}),(\overline{(a, i)} \rightarrow \lambda) \mid(a, i) \in F\} \\
& \left.\cup\{\overline{(a, i)} \rightarrow \overline{(a, i)}, j \rightarrow \lambda) \mid(a, i) \in F, i \in H^{\prime}, j \in H\right\} \\
& \left.\cup\{\overline{(a, s)} \rightarrow \overline{(a, s)}, j \rightarrow \lambda) \mid(a, s) \in F, j \in H-H_{a}\right\} .
\end{aligned}
$$

The set $F \subset O \times H$ is defined in such a way that a pair $(a, i)$ is in $F$ if and only if no rules of region $i$ are applicable to $a$, eventually except for rules of the form $a \rightarrow b_{i n_{j}} u_{\text {out }}$ in $R_{1}$, and for any $a \in O, H_{a} \subset H^{\prime}$ denotes the set of membrane labels $j$ such that $R_{s}$ contains rules of the form $a \rightarrow b_{i n_{j}} u_{\text {out }}, a, b \in O, u \in O^{*}$. We can now see that a configuration with object $a$ in region $i$ is a halting one if and only if $(a, i) \in F$, and (i) either $i \neq s$, or (ii) $i=s$ and no membranes with labels from $H_{a}$ are present. The rules with the barred symbols $\overline{(a, i)}$ allow us to remove all non-terminals from the sentential form of $M$ when $\Pi$ has reached a halting configuration. Hence, $\operatorname{Ps}(L(M))$ equals the set of vectors generated by $\Pi$.

To show the converse inclusion, we consider a matrix grammar $G=(N, T, S, M)$ without appearance checking in the $f$-binary normal form and construct a $\mathrm{P}$ system $\Pi$ simulating $G$ as follows: $\Pi$ starts with the initial configuration $\left[{ }_{s} S\right]_{s}$.

$$
\begin{aligned}
\Pi= & \left(O, H,\left[_{s}\right]_{s}, S, R_{A_{\text {init }}}, \ldots, R_{s}\right), \\
O= & \left\{S, S_{1}, f, \#\right\} \cup N_{1} \cup\left\{l_{i} \mid l \in M, 0 \leq i \leq 4\right\}, \\
H= & N_{2} \cup\{s\}, \\
R_{s}= & \left\{S \rightarrow\left[A_{\text {init }} S_{1}\right]_{A_{\text {init }}} \mid\left(S \rightarrow X_{\text {init }} A_{\text {init }}\right) \in M\right\} \\
& \cup\left\{X \rightarrow\left(l_{0}\right)_{\text {in }_{A}} \mid l:(X \rightarrow Y, A \rightarrow u v) \in M\right\} \\
& \cup\left\{l_{1} \rightarrow\left[{ }_{u} l_{2}\right]_{u} \mid l:(X \rightarrow Y, A \rightarrow u v) \in M, u \in N_{2}\right\} \\
& \cup\left\{l_{1} \rightarrow l_{3} u_{\text {out }} \mid l:(X \rightarrow Y, A \rightarrow u v) \in M, u \in T \cup\{\lambda\}\right\}
\end{aligned}
$$




$$
\begin{aligned}
& \cup\left\{l_{3} \rightarrow\left[{ }_{v} l_{4}\right]_{v} \mid l:(X \rightarrow Y, A \rightarrow u v) \in M, v \in N_{2}\right\} \\
& \cup\left\{l_{3} \rightarrow Y v_{\text {out }} \mid l:(X \rightarrow Y, A \rightarrow u v) \in M, v \in T \cup\{\lambda\}\right\} \\
& \cup\left\{X \rightarrow \#, \mid X \in N_{1} \cup\{\#\} \cup\left\{f \rightarrow \#_{\text {in }_{A}} \mid A \in N_{2}\right\},\right. \\
R_{A}= & \left\{S_{1} \rightarrow\left(X_{\text {init }}\right)_{\text {out }} \mid\left(S \rightarrow X_{\text {init }} A\right) \in M\right\} \\
& \cup\left\{l_{0} \rightarrow l_{1} \delta \mid l:(X \rightarrow Y, A \rightarrow u v) \in M\right\} \\
& \cup\left\{l_{2} \rightarrow\left(l_{3}\right)_{\text {out }} \mid l:(X \rightarrow Y, B \rightarrow A v) \in M\right\} \\
& \cup\left\{l_{4} \rightarrow(Y)_{\text {out }} \mid l:(X \rightarrow Y, B \rightarrow u A) \in M\right\} \cup\{\rightarrow \#\} .
\end{aligned}
$$

The rules $S \rightarrow\left[{ }_{A_{\text {init }}} S_{1}\right]_{A_{\text {init }}} \in R_{S}$ as well as $S_{1} \rightarrow\left(X_{\text {init }}\right)_{\text {out }} \in R_{A_{\text {init }}}$ simulate the start matrix by producing a membrane corresponding to the literal symbol $A_{\text {init }}$ and an object corresponding to the control symbol $X_{\text {init }}$. A matrix $l:(X \rightarrow Y, A \rightarrow u v)$ is simulated as follows: first, object $X$ removes a membrane with label $A$ by the rules $X \rightarrow\left(l_{0}\right)_{i n_{A}} \in R_{S}$ and $l_{0} \rightarrow l_{1} \delta \in R_{A}$; then, it creates membranes with labels $u, v$ if $u, v$ are non-terminal symbols, or sends $u, v$ into the environment if $u, v$ are terminal symbols. Finally, the object $X$ changes to $Y$. The rules $X \rightarrow \#, \# \rightarrow \# \in R_{S}$ guarantee that, if the derivation of $G$ is 'stuck' in a form which is not terminal, then the corresponding computation in $\Pi$ will enter an infinite loop. Finally, the result of a derivation in $G$ is terminal if and only if $f$ is produced and no other non-terminals have remained; this is checked by $f \rightarrow \#_{i n_{A}} \in R_{S}$ (after the application of such a rule, $\# \rightarrow \# \in R_{A}$ then guarantees that the corresponding computation in $\Pi$ will enter an infinite loop). Observing that the above P system fulfills all the conditions stated in the theorem concludes the proof.

We conjecture that we need not restrict the membrane structure, i.e. $P S M A T^{\lambda}=$ Ps $O_{1,1, *} P_{*, *, *}($ ncoo, tar, mcre,$\delta)$.

\subsection{Number of symbols}

The cardinality of the alphabet in the computational completeness proofs usually depends on the complexity parameters of the simulated device. We will now show that any recursively enumerable set of $m$-dimensional vectors of non-negative integers can be generated by $\mathrm{P}$ systems with membrane creation and dissolution, having an alphabet of $10+m$ symbols.

THEOREM $3.4 L(m) O_{1,2,10+m} P_{2, *, *}($ ncoo, tar, mcre,$\delta)=R E(m)$.

Proof We simulate a register machine $M=\left(2, T, P, l_{0}, l_{h}\right)$, where $T=\left\{a_{i} \mid 1 \leq i \leq m\right\}$; let $P_{+}$denote the set of all ADD instruction labels, and let $P_{-}$denote the set of all SUB instruction labels.

$$
\begin{aligned}
\Pi & =\left(O, H,\left[s[I]_{I}\right]_{s}, \lambda, b, R_{l_{0}}, \ldots, R_{D}\right), \\
O & =T \cup\{b, c, d, e\} \cup\left\{r_{+}, r_{-}, r_{-}^{\prime} \mid r \in\{1,2\}\right\}, \\
H & =P \cup\{I, 1,2, s, D\}, \\
R_{i} & =R_{i, I} \cup R_{i, O} \cup R_{i, A} \cup R_{i, S} \cup R_{i, D} \cup R_{i, Z} \cup R_{i, N}, i \in H .
\end{aligned}
$$

For clarity, the rules are grouped (initialization, output, add, subtract, decrement case, zero case, next instruction). 


\section{Initialization:}

$$
\begin{aligned}
R_{I, I} & =\left\{b \rightarrow\left[l_{l_{0}} d\right]_{l_{0}}\right\}, \\
R_{i, I} & =\emptyset, i \in H-\{I\} .
\end{aligned}
$$

\section{Output:}

$$
\begin{aligned}
R_{l, O}= & \left.a \rightarrow a_{\text {out }} \mid l:\left(\text { write }(a), l^{\prime}\right), a \in T\right\} \\
& \cup\{a \rightarrow a \delta \mid a \in T\}, l \in P \cup\{I\}, \\
R_{s, O}= & \left\{a \rightarrow a_{\text {out }} b, a \rightarrow a_{\text {out }} c \mid a \in T\right\}, \\
R_{i, O}= & \emptyset, i \in H-(P \cup\{I, s\}) .
\end{aligned}
$$

Add:

$$
\begin{aligned}
R_{l, A}= & \left\{d \rightarrow\left(r_{+}\right)_{\text {out }} \mid l:\left(A(r), l^{\prime}, l^{\prime \prime}\right) \in P, r \in\{1,2\}\right\} \\
& \cup\left\{r_{+} \rightarrow r_{+} \delta \mid r \in\{1,2\}\right\}, l \in P \cup\{I\}, \\
R_{s, A}= & \left\{r_{+} \rightarrow\left[{ }_{r} d\right]_{r} \mid r \in\{1,2\}\right\}, \\
R_{r, A}= & \left\{d \rightarrow b_{\text {out }}, d \rightarrow c_{\text {out }}\right\}, r \in\{1,2\}, \\
R_{D, A}= & \emptyset .
\end{aligned}
$$

\section{Subtract:}

$$
\begin{aligned}
R_{l, S}= & \left\{d \rightarrow\left(r_{-}\right)_{\text {out }} \mid l:\left(S(r), l^{\prime}, l^{\prime \prime}\right) \in P, r \in\{1,2\}\right\} \\
& \cup\left\{r_{-} \rightarrow r_{-} \delta \mid r \in\{1,2\}\right\}, l \in P \cup\{I\}, \\
R_{s, S}= & \left\{r_{-} \rightarrow r_{-}^{\prime} d, d \rightarrow\left[{ }_{D} d\right]_{D} \mid r \in\{1,2\}\right\}, \\
R_{D, S}= & \{d \rightarrow \lambda\}, \\
R_{i, S}= & \emptyset, i \in H-P-\{I, s, D\} .
\end{aligned}
$$

\section{Decrement case:}

$$
\begin{aligned}
R_{s, D} & =\left\{r_{-}^{\prime} \rightarrow\left(r_{-}^{\prime}\right)_{i n_{r}}, e \rightarrow e_{i n_{D}} \mid r \in\{1,2\}\right\}, \\
R_{r, D} & =\left\{r_{-} \rightarrow r_{-}^{\prime} d, d \rightarrow[d d]_{D}\right\}, r \in\{1,2\}, \\
R_{D, D} & =\left\{r_{-}^{\prime} \rightarrow e_{\text {out }}\right\}, r \in\{1,2\}, \\
R_{i, D} & =\emptyset, i \in H-\{1,2, s, D\} .
\end{aligned}
$$

\section{Zero case:}

$$
\begin{aligned}
R_{s, Z} & \left.=\left\{r_{-}^{\prime} \rightarrow\left(r_{-}^{\prime}\right)_{i n_{D}}\right\} \mid r \in\{1,2\}\right\}, \\
R_{D, Z} & =\left\{r_{-}^{\prime} \rightarrow b \delta \mid r \in\{1,2\}\right\}, \\
R_{i, D} & =\emptyset, i \in H-\{s, D\} .
\end{aligned}
$$

\section{Next instruction:}

$$
\begin{aligned}
& R_{s, N}=\left\{b \rightarrow b_{i n_{l}}, c \rightarrow c_{i n_{l}} \mid l \in P\right\}, \\
& R_{l, N}=\left\{b \rightarrow\left[l^{\prime} a l_{l^{\prime}}, c \rightarrow\left[l_{l^{\prime \prime}} a\right]_{l^{\prime \prime}} \mid l:\left(X(r), l^{\prime}, l^{\prime \prime}\right) \in P, X \in\{A, S\}\right\} .\right.
\end{aligned}
$$

The instruction labels are encoded into membrane labels, and the values of the registers are encoded by the number of copies of membranes associated with them. The proof mainly relies 
on the fact that the amount of information needed to be transmitted between the instructions and the registers is 'small', i.e. the instructions tell us which operation (ADD or SUB, represented by $\left.r_{+}, r_{-}, r \in\{1,2\}\right)$ has to be applied and which register $r$ it has to be applied to. The objects $r_{-}^{\prime}, r \in\{1,2\}$, and $e$ are used to implement the SUB instruction, and the object $d$ is used here to organize a delay for appearance checking, similar to the technique from theorem 3.1, whereas otherwise it is used when the membranes already contain all the information needed.

After an operation has been simulated, the next instruction is chosen from two variants, non-deterministically chosen in the ADD case and, as in the SUB case, depending here on whether decrementing has been successful or not. These variants are represented by the objects $b, c$. The transition to the next instruction is done in the following way. Object $b$ in membrane $l$ creates membrane $l^{\prime}$, or object $c$ in membrane $l$ creates membrane $l^{\prime \prime}$. Then, the object 'memorizes' the next register to be operated on and the operation to be performed, and then membrane $l$ is dissolved, leaving the newly created membrane in the skin.

If we want to start with the simplest membrane structure, one more symbol is needed as shown in the following.

THEOREM $3.5 L(m) O_{1,2,11+m} P_{1, *, *}($ ncoo, tar, mcre,$\delta)=R E(m)$.

Proof We again simulate a register machine $M=\left(2, T, P, l_{0}, l_{h}\right)$ as in the proof of the preceding theorem, but in the $\mathrm{P}$ system $\Pi^{\prime}$ we use an additional symbol $a$ for an initial step starting in the skin membrane:

$$
\begin{aligned}
\Pi^{\prime} & =\left(O, H,\left[_{s}\right]_{s}, a, R_{l_{0}}, \ldots, R_{D}\right), \\
O & =T \cup\{a, b, c, d, e\} \cup\left\{r_{+}, r_{-}, r_{-}^{\prime} \mid r \in\{1,2\}\right\}, \\
H & =P \cup\{I, 1,2, s, D\}, \\
R_{i} & =R_{i, I} \cup R_{i, O} \cup R_{i, A} \cup R_{i, S} \cup R_{i, D} \cup R_{i, Z} \cup R_{i, N}, i \in H .
\end{aligned}
$$

\section{Initialization:}

$$
\begin{aligned}
& R_{s, I}=\left\{a \rightarrow\left[{ }_{I} b\right]_{I}\right\}, \\
& R_{I, I}=\left\{b \rightarrow\left[l_{0} d\right]_{l_{0}}\right\}, \\
& R_{i, I}=\emptyset, i \in H-\{s, I\} .
\end{aligned}
$$

Except for the initialization, the sets of rules are exactly the same as for the P system $\Pi$ constructed in the preceding proof, an observation that completes this proof.

\section{One polarization}

The theorem below provides a result, similar to that of theorem 3.1, for P systems with active membranes with only one polarization (usually called $P$ systems with active membranes without polarizations). The construction gives no upper bound on the number of objects present inside the system in general, but during any halting computation the number of objects never exceeds three.

THEOREM 4.1 L $O_{1,3 / *, *} P_{4, *, 3}$ active $\left._{1}, a, b, c, d, e\right)=R E$. 
Proof In the description of the $\mathrm{P}$ system $\Pi$ below, $w_{s}$ describes the initial multiset for the skin membrane, whereas $w_{s}^{\prime}$ denotes the initial multiset in the elementary membrane having the same label as the skin membrane. We now simulate a register machine $M=\left(2, T, P, l_{0}, l_{h}\right)$ :

$$
\begin{aligned}
\Pi & =\left(O, \mu, w_{s}, w_{1}, w_{2}, w_{s}^{\prime}, R\right), \\
\mu & =\left[{ }_{s}[1]_{1}[2]_{2}[s]_{s}\right]_{s}, \\
O & =T \cup\left\{a_{i} \mid 1 \leq i \leq 2\right\} \cup\left\{l, l_{1}, l_{2} \mid l \in P\right\} \cup\left\{b_{1}, b_{2}, t, d, \#\right\}, \\
w_{s} & =l_{0}, w_{1}=w_{2}=w_{s}^{\prime}=\lambda, \\
R & =R_{O} \cup R_{A} \cup R_{S} \cup R_{D} \cup R_{Z} .
\end{aligned}
$$

The rules are grouped into categories: output, add, subtract, decrement case and zero case.

\section{Output:}

$$
R_{O}=\left\{\left[l \rightarrow l^{\prime} a\right]_{s}, \quad\left[l \rightarrow l^{\prime \prime} a\right]_{s},[a]_{s} \rightarrow[]_{s} a \mid l:\left(\text { write }(a), l^{\prime}\right), a \in T\right\} .
$$

Add:

$$
\begin{aligned}
R_{A}= & \left\{l[]_{i} \rightarrow[l]_{i},[l]_{i} \rightarrow\left[l_{1}\right]_{i}[t]_{i},[t \rightarrow \lambda]_{i},\left[l_{1}\right]_{i} \rightarrow[]_{i} l^{\prime},\right. \\
& {\left.\left[l_{1}\right]_{i} \rightarrow[]_{i} l^{\prime \prime} \mid l:\left(A(i), l^{\prime}, l^{\prime \prime}\right), i \in\{1,2\}\right\} . }
\end{aligned}
$$

\section{Subtract:}

$$
\begin{aligned}
R_{S}= & \left\{\left[l \rightarrow d b_{i} l_{1}\right]_{s}, b_{i}[]_{i} \rightarrow\left[b_{i}\right]_{i},\left[b_{i}\right]_{i} \rightarrow[]_{i} t,[t \rightarrow \lambda]_{s},\right. \\
& {\left.\left[b_{i} \rightarrow \#\right]_{s},\left[b_{i} \rightarrow \#\right]_{i},[\# \rightarrow \#]_{i} \mid l:\left(S(i), l^{\prime}, l^{\prime \prime}\right), i \in\{1,2\}\right\} } \\
& \cup\left\{d[]_{s} \rightarrow[t]_{s},[t \rightarrow \lambda]_{s}[d \rightarrow \#]_{s},[\# \rightarrow \#]_{s}\right\} .
\end{aligned}
$$

\section{Decrement case:}

$$
R_{D}=\left\{l_{1}[]_{i} \rightarrow\left[l_{1}\right]_{i},\left[l_{1}\right]_{i} \rightarrow l^{\prime} \mid l:\left(S(i), l^{\prime}, l^{\prime \prime}\right), i \in\{1,2\}\right\} .
$$

\section{Zero case:}

$$
R_{Z}=\left\{l_{1}[]_{s} \rightarrow\left[l_{2}\right]_{s},\left[l_{2}\right]_{s} \rightarrow[]_{s} l^{\prime \prime} \mid l \in P_{-}\right\} .
$$

As in the previous theorem, we simulate a register machine with output tape and two registers; the values of registers $i, i \in\{1,2\}$, are represented by the multiplicities of membranes $i$. However, since new membranes can only be created by dividing existing ones, one extra membrane is needed for every register. The duty of $d$ is to 'keep busy' the elementary membrane with label $s$ (otherwise \# appears and the computation does not halt), and the use of the objects $b_{i}$ is to 'keep busy' one membrane with label $i$ for two steps. Object $t$ is not needed for the computation, it is only used to keep the usual form of the membrane division and communication rules; it is immediately erased.

Generating an output is done by a non-cooperative rule changing the instruction label and producing the corresponding symbol, which is then sent out. Incrementing a register $\left(l:\left(A(i), l^{\prime}, l^{\prime \prime}\right)\right)$ is done in the following way: $l$ enters membrane $i$ (there is always at least one), dividing it. The object $l_{1}$ in one copy is sent to the skin as $l^{\prime}$ or $l^{\prime \prime}$, while the object $t$ in the other copy is erased.

Subtracting with $\left(l:\left(S(i), l^{\prime}, l^{\prime \prime}\right)\right)$ is done by keeping busy the elementary membrane with label $s$ for one step and one membrane with label $i$ for two steps, while object $l_{1}$ tries to enter 
Theorem 4.1

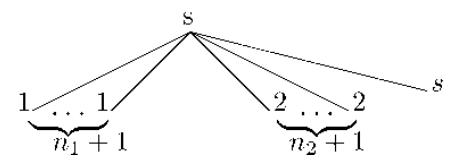

Theorem 4.2

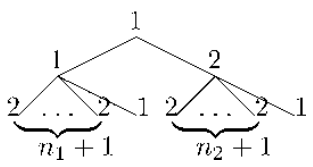

Figure 2. Membrane structures for the active membrane proofs.

any membrane with label $i$. If the register is not zero, then $l_{1}$ immediately enters one of the other membranes with label $i$, dissolves it and changes to $l^{\prime}$. Otherwise, after waiting for one step, object $l_{1}$ enters the elementary membrane with label $s$ and returns to the skin as $l^{\prime \prime}$.

During a correct simulation of a run of the register machine (in particular, during any halting computation) there are never more than three objects present inside the system.

It is possible to reduce the number of membrane labels to two at the price of starting with seven membranes.

TheOREM $4.2 L O_{1,3 / *, *} P_{7, *, 2}$ (active $\left._{1}, a, b, c, d, e\right)=R E$.

Proof (sketch) Similarly to the proof of theorem 3.1, let us start with a membrane structure $\left.{ }_{1}\left[1[1]_{1}[2]_{2}\right]_{1}\left[2\left[l_{1}\right]_{1}[2]_{2}\right]_{2}\right]_{1}$ (see also figure 2 ), and represent the values of a working register $i$ by the number of elementary membranes with label 2, inside the membranes with labels $i$, minus one. The elementary membranes with label 1 will be used for delay, just like the elementary membrane labeled $s$ was used in the proof of theorem 4.1, and the instructions are simulated accordingly. The main difference is that when simulating an ADD or a SUB instruction, we have one additional initial step at the beginning choosing the 'cluster membrane' representing the corresponding register (see the proof of theorem 3.1). Obviously, at the end of the simulation of the instruction in the right 'cluster membrane', we need an additional final step for moving the instruction label back to the skin membrane.

\section{Two polarizations}

The next theorem shows that, with two polarizations, we need only one membrane to simulate register machines in a deterministic way.

THEOREM 5.1 DPs $s_{a} O P_{1,1,1}\left(\right.$ active $\left._{2}, a, c\right)=P S R E$.

Proof We will simulate the actions of a deterministic register machine $M=\left(d, P, l_{0}, l_{h}\right)$ with $d$ registers by a deterministic $\mathrm{P}$ system with one membrane and two polarizations. For every instruction $l$, let us denote that register $l$ acts by $r(l)$ and that operation $l$ is carried out by $o p(l)$.

$$
\begin{aligned}
\Pi= & \left(O, E,[1]_{1}^{0}, w_{1}, R\right), \\
O= & \{(a, i, j) \mid 1 \leq i \leq d, 0 \leq j \leq d+2\} \\
& \cup\{(l, i, j) \mid l \in P, 0 \leq i \leq 2,1 \leq j \leq d+2\} \cup\{\#\}, \\
E= & \{0,1\} \\
w_{1}= & \left(l_{0}, 0,0\right) .
\end{aligned}
$$


The system receives the input $(a, 1,0)^{n_{1}} \ldots(a, d, 0)^{n_{d}}$ in addition to $w_{1}$ in the skin. The set $R$ contains the rules

$$
\begin{aligned}
& {[z]_{1}^{e} \rightarrow[]_{1}^{1-e} z, \quad e \in\{0,1\},} \\
& {[(a, i, j) \rightarrow(a, i, j+1)]_{1}^{0}, \quad 1 \leq i \leq d, 0 \leq j \leq d+1,} \\
& {[(a, i, d+2) \rightarrow(a, i, 0)]_{1}^{0}, \quad 1 \leq i \leq d,} \\
& {[(a, i, j+1)]_{1}^{1} \rightarrow[]_{1}^{0}(a, i, j+1), \quad 1 \leq i \leq d,} \\
& {[(l, 0, j) \rightarrow(l, 0, j+1)]_{1}^{0}, \quad l \in P, 0 \leq j<r(l)-1,} \\
& {[(l, i, j) \rightarrow(l, i, j+1)]_{1}^{0}, \quad l \in P, i \in\{1,2\}, r(l) \leq j \leq d+1,} \\
& {\left[(l, 1, d+2) \rightarrow\left(l^{\prime}, 0,0\right)\right]_{1}^{0}, \quad l \in P,} \\
& {\left[(l, 2, d+2) \rightarrow\left(l^{\prime \prime}, 0,0\right)\right]_{1}^{0}, \quad l \in P,} \\
& {[(l, 0, j) \rightarrow(l, 1, j+1)(a, j+1, j+1)]_{1}^{0}, \quad l \in P,} \\
& \quad j=r(l)-1, o p(l)=A, \\
& {[(l, 0, j) \rightarrow(l, 0, j+1) z]_{1}^{0}, \quad l \in P, j=r(l)-1, \text { op }(l)=S,} \\
& {[(l, 0, j) \rightarrow(l, 0, j+1)]_{1}^{0}, \quad l \in P, j=r(l), \text { op }(l)=S,} \\
& {[(l, 0, j) \rightarrow(l, 0, j+1)]_{1}^{1}, \quad l \in P, j=r(l)+1, \operatorname{op}(l)=S,} \\
& {[(l, 0, j) \rightarrow(l, 1, j+1)]_{1}^{0}, \quad l \in P, j=r(l)+2, \operatorname{op}(l)=S,} \\
& {[(l, 0, j) \rightarrow(l, 2, j) z]_{1}^{1}, \quad l \in P, j=r(l)+2, \operatorname{op}(l)=S,} \\
& {[(l, 2, j) \rightarrow(l, 2, j+1)]_{1}^{1}, \quad l \in P, j=r(l), \operatorname{op}(l)=S,} \\
& {\left[\left(l_{h}, 0,0\right)\right]_{1}^{0} \rightarrow[]_{1}^{1}\left(l_{h}, 0,0\right), \quad 1 \leq i \leq d .}
\end{aligned}
$$

The idea of this proof is similar to that from $[5,6]$ : the symbols corresponding to the registers have states (second subscript) $0, \ldots, d+2$, and so do the symbols corresponding to the instructions of the register machine. The first subscript of the instruction symbols is 0 if the instruction has not yet been applied, it is 1 if increment or decrement has been applied, and 2 if the decrement has failed.

Most of the time the polarization is 0 ; object $z$ can reverse the polarization by (1). When the polarization is 0 , the register symbols cycle through the states by (2), (3). Before the current instruction $l$ is applied, the instruction symbols also cycle through the states until the state becomes $r(l)-1$, i.e. the index of the register (the instruction operates on) minus one. We will explain the details of the application below. After the instruction has been applied, the first subscript of the instruction symbol changes to 1 or 2 and it cycles through the states by (6), finally changing into $l^{\prime}$ by (7) or into $l^{\prime \prime}$ by (8).

Addition is done by rule (9). Decrement is done by a 'diagonalization technique': polarization 1 when register $i$ is in state $i+1$ signals a decrement attempt of register $i$ by (4), and the polarization will change if and only if it has been successful. Thus, to apply $l:\left(S(i), l^{\prime}, l^{\prime \prime}\right)$, the instruction symbol in state $i-1$ additionally produces a symbol $z$. By the time $z$ changes the polarization to 1 by (1), all other symbols reach state $i+1$. After one more step the state symbol checks whether the decrement has been successful, (13), or not, (14). After a successful decrement, all symbols continue changing states with polarization 0 and state $i+1$. Otherwise, the instruction symbol additionally produces a symbol $z$ and, after one more step, all symbols continue changing states with polarization 0 and state $i+1$. 
After the simulation of $M$ having reached the final label, the instruction symbol exits the system, changing the polarization to 1 . Since the register symbols are in state 1 , the system halts.

Looking into the proof of the preceding theorem we realize that even a more general result is shown: the multisets remaining in the skin membrane at the end of a halting computation can be interpreted as the computation result:

COROLlary 5.2 Any partially recursive function can be computed by a deterministic $P$ system with one membrane, two polarizations and internal output.

\section{Restricted membrane creation}

We now revisit the membrane creation case and consider restricted membrane creation: in region $i$ it is only possible to create membranes with label $i$.

THEOREM $6.1 \quad P_{s} O_{1,1, *} P_{*, *, *}\left(\right.$ ncoo, tar, mcre $\left._{r}, \delta\right) \supseteq P s M A T^{\lambda}$.

Proof This theorem follows the same line as the second part of theorem 3.3 (see also the explanations given there). We simulate a matrix grammar $G=(N, T, S, M)$ in the $f$-binary normal form. The multiplicity of any 'literal non-terminal' $A \in N_{2}$ (see the definition) is represented by the number of membranes with label $A$. However, these membranes are not directly in the skin membrane, but rather inside a cluster membrane with label $A$, which is in the skin. Let us refer to the 'literal non-terminals' $N_{2}$ as $\left\{A_{i} \mid 1 \leq i \leq n\right\}$, and let $A_{\text {init }}=A_{1}$.

$$
\begin{aligned}
\Pi & =\left(O, H, \mu, w_{s}, w_{A_{1}}, \ldots, w_{A_{n}}, R_{s}, R_{A_{1}}, \ldots, R_{A_{n}}\right), \\
O & =\left\{S, S_{1}, f, \#\right\} \cup N_{1} \cup\left\{l_{i} \mid l \in M, 0 \leq i \leq 5\right\} \cup\left\{f_{i} \mid 1 \leq i \leq n+1\right\}, \\
H & =\{s\} \cup N_{2}, \\
\mu & =\left[s\left[{ }_{A_{1}}\right]_{A_{1}} \ldots\left[\left[_{A_{n}}\right]_{A_{n}}\right]_{S},\right. \\
w_{s} & =S, \\
w_{A} & =\lambda, A \in N_{2} .
\end{aligned}
$$

The $\mathrm{P}$ system $\Pi$ now simulates the actions of $G$ using the following rules.

Start $\left(S \rightarrow X_{\text {init }} A_{1}\right) \in M$ :

$$
\begin{aligned}
& S \rightarrow(S)_{A_{1}} \in R_{s}, \\
& S \rightarrow\left[{ }_{A_{1}} S_{1}\right]_{A_{1}}, S_{1} \rightarrow\left(S_{1}\right)_{\text {out }} \in R_{A_{1}}, \\
& S_{1} \rightarrow X_{\text {init }} \in R_{s} .
\end{aligned}
$$


Applying matrix $l:(X \rightarrow Y, A \rightarrow u v) \in M, u, v \in N_{2} \cup T \cup\{\lambda\}$ :

$$
\begin{aligned}
& X \rightarrow(X)_{\text {in }_{A}} \in R_{s}, \\
& X \rightarrow \#, \# \rightarrow \# \in R_{A}, \\
& X \rightarrow\left(l_{0}\right)_{\text {in }_{A}}, l_{0} \rightarrow l_{1} \delta, l_{1} \rightarrow\left(l_{1}\right)_{\text {out }} \in R_{A}, \\
& l_{1} \rightarrow l_{3} u_{\text {out }} \in R_{s}, \text { if } u \in T \cup\{\lambda\}, \\
& l_{1} \rightarrow\left(l_{2}\right)_{\text {in }} \in R_{s}, \text { if } u \in N_{2}, \\
& l_{2} \rightarrow\left[{ }_{u} l_{3}\right]_{u}, l_{3} \rightarrow\left(l_{3}\right)_{\text {out }} \in R_{u}, \\
& l_{3} \rightarrow l_{5} v_{\text {out }} \in R_{s}, \text { if } v \in T \cup\{\lambda\}, \\
& l_{3} \rightarrow\left(l_{4}\right)_{\text {inv }} \in R_{s}, \text { if } v \in N_{2}, \\
& l_{4} \rightarrow\left[{ }_{v} l_{5}\right]_{v}, l_{5} \rightarrow\left(l_{5}\right)_{\text {out }} \in R_{v}, l_{5} \rightarrow Y \in R_{s} .
\end{aligned}
$$

\section{Finish:}

$$
\begin{aligned}
& f \rightarrow f_{1} \in R_{s}, \\
& f_{i} \rightarrow\left(f_{i}\right)_{i n_{A_{i}}} \in R_{s}, \quad 1 \leq i \leq n, \\
& f_{i} \rightarrow f_{i+1} \delta \in R_{A_{i}}, \quad 1 \leq i \leq n \\
& f_{n+1} \rightarrow(\#)_{i n_{A}} \in R_{s}, A \in N_{2}, \\
& \# \rightarrow \# \in R_{A}, A \in N_{2} .
\end{aligned}
$$

Information concerning the 'control non-terminal' $X \in N_{1}$ (see the definition of matrix grammars in $f$-binary normal form) is stored in only one object that travels across the membrane structure, updating the membrane structure and itself according to the matrices of $G$. At the end of the simulation of $G$, the object dissolves all cluster membranes and finally tries to enter any remaining membrane and thus start an infinite loop. Hence, the computation halts if and only if a terminal form has been reached.

\section{Conclusions and open problems}

We have shown that $\mathrm{P}$ systems with membrane creation generate $R E$, using two membrane labels and at most two objects present inside the system throughout the computation. Accepting any recursively enumerable language can also be done with two membrane labels. On the other hand, it is possible to bound the number of symbols by $m+10$ and still generate $R E(m)$, provided that the number of membrane labels is unbounded.

We have also shown that $R E$ is generated by $\mathrm{P}$ systems using four membranes and three labels or seven membranes and two labels in the initial configuration, where at most three objects are ever present in any halting computation.

It is known from $[5,6]$ that $P$ systems with two polarizations and rules of types (a) and (c) generate $P S R E$ using two membranes, or accept $P S R E$ using one membrane. We have proved in this article that deterministic systems of this kind with one membrane accept PsRE. Moreover, in the proof of this result (theorem 5.1), the rules are global (there is only one membrane) and rules of type (c) are non-renaming (the contents of the environment do not matter). 
Improving any complexity parameter greater than one (especially in the case of $*$ ) in any theorem is an open question. Moreover, the following questions are of interest.

- What is the power of $\mathrm{P}$ systems with membrane creation and one object?

- What is the power of deterministic P systems with membrane division (without polarizations, without changing labels, etc.)?

- What is the exact power of P systems with restricted membrane creation?

- How can the types of rules be restricted in theorem 4.1?

- How can target indications be restricted in theorem 3.1?

- What further restrictions cause a complexity trade-off?

- What is the generative power of $\mathrm{P}$ systems without polarizations and $m$ membranes, $m=$ $1,2,3$ ?

- What is the generative power of one-membrane P systems with two polarizations and external output?

\section{Acknowledgements}

The first author is supported by the project TIC2002-04220-C03-02 of the Research Group on Mathematical Linguistics, Tarragona, and acknowledges the Moldovan Research and Development Association (MRDA) and the U.S. Civilian Research and Development Foundation (CRDF), award No. MM2-3034. The third author would like to acknowledge the support from Ministerio de Ciencia y Tecnología of Spain, through Plan Nacional de $I+D+I$ (2000-2003) (TIC2002-04220-C03-01), co-financed by FEDER funds.

\section{References}

[1] Păun, Gh., 2000, Computing with membranes. Journal of Computer and System Sciences, 61, 108-143; TUCS Research Report 208, 1998. Available online at: http://www.tucs.fi.

[2] Păun, Gh., 2002, Membrane Computing: An Introduction (Berlin: Springer).

[3] The P systems webpage. Available online at: psystems.disco.unimib.it (accessed December 2005).

[4] Alhazov, A., 2004, P systems without multiplicities of symbol-objects. Information Processing Letters, submitted (preprint version: www.geocities.com/aartiom).

[5] Alhazov, A., Freund, R. and Păun, Gh., 2005, In M. Margenstern (Ed.) Machines, Computations, and Universality, International Conference, MCU 2004, Saint Petersburg, 2004, Lecture Notes in Computer Science 3354 (Berlin: Springer).

[6] Alhazov, A., Freund, R. and Păun, Gh., 2004, In Gh. Păun, A. Riscos-Núñez, A. Romero-Jiménez and F. SanchoCaparrini (Eds) Second Brainstorming Week on Membrane Computing, RGNC Technical Report 01/2004 (Seville: University of Seville).

[7] Dassow, J. and Păun, Gh., 1989, Regulated Rewriting in Formal Language Theory (Berlin: Springer).

[8] Salomaa, A. and Rozenberg, G. (Eds), 1997, Handbook of Formal Languages (Berlin: Springer).

[9] Minsky, M., 1967, Computation. Finite and Infinite Machines (Englewood Cliffs, NJ: Prentice Hall). 